\title{
Commentary: The laws of robotics
}

\author{
Moritz C. Wyler von Ballmoos, MD, and \\ Michael J. Reardon, MD
}

First Law:

A robot may not injure a human being or, through inaction, allow a human being to come to harm. Second Law:

A robot must obey the orders given it by human beings except where such orders would conflict with the

First Law.

Third Law:

A robot must protect its own existence as long as such protection does not conflict with the First or Second Law

$$
\text { -Isaac Asimov }
$$

The surgical treatment of symptomatic severe aortic stenosis (AS) with surgical aortic valve replacement (SAVR) can be easily designated as one of the great medical triumphs of the 20th century. The 21st century has brought us an increasing variety of less-invasive ways to treat cardiovascular diseases, and AS is no exception. Transcatheter aortic valve replacement (TAVR), which was initially seen as an option for those who were either not or at least very poor candidates for SAVR, was first successfully accomplished by Cribier and colleagues ${ }^{1}$ in 2002. In less than 2 decades, TAVR volume has exploded and transformed the treatment paradigm for severe AS. There are now more cases of TAVR for severe AS than all surgical cases combined. This growth was built largely on the data from 2 families of randomized trials comparing TAVR with SAVR. Both high-risk randomized trials used all-cause mortality as their primary end point and found TAVR to be either noninferior ${ }^{2}$ or superior $^{3}$ to SAVR at 1 year. Both intermediate-risk trials used allcause mortality or disabling stroke as their primary end points and found TAVR to be noninferior to SAVR at

From the Department of Cardiovascular Surgery, Houston Methodist Hospital, Houston, Tex.

Disclosures: The authors reported no conflicts of interest.

The Journal policy requires editors and reviewers to disclose conflicts of interest and to decline handling or reviewing manuscripts for which they may have a conflict of interest. The editors and reviewers of this article have no conflicts of interest.

Received for publication Oct 27, 2020; revisions received Oct 27, 2020; accepted for publication Oct 28, 2020; available ahead of print Nov 5, 2020.

Address for reprints: Michael J. Reardon, MD, Department of Cardiovascular Surgery, Houston Methodist Hospital, 6550 Fannin, Suite 1401, Houston, TX 7030 (E-mail: mreadon@houstonmethodist.org).

J Thorac Cardiovasc Surg 2021;161:1760-1

$0022-5223 / \$ 36.00$

Copyright (c) 2020 by The American Association for Thoracic Surgery

https://doi.org/10.1016/j.jtcvs.2020.10.112



CENTRAL MESSAGE

A successful robotic AVR team requires preprocedure training, a dedicated and consistent team, and transparent reporting of results.

2 years. ${ }^{4,5}$ The low-risk trials deviated in their primary end points. One used all-cause mortality, stroke, or rehospitalization at 1 year and showed TAVR to be superior to SAVR. ${ }^{6}$ The other used the more conservative all-cause mortality or disabling stoke at 2 years and found TAVR to be noninferior to SAVR. ${ }^{7}$ These outstanding results pose a challenge to cardiac surgeons to explain why SAVR might be a preferred option.

In this issue of the Journal, Badhwar and colleagues ${ }^{8}$ beautifully detail their institutional experience developing and implementing a robotic surgical aortic valve replacement (rAVR) program. They developed the technical aspects in the cadaver laboratory over a 24 -month period under the guidance of 2 dedicated surgeons. Once they were comfortable with the procedure in the laboratory, they took their knowledge to the operating room and performed 20 consecutive fully robotic rAVRs between January 2020 and July 2020, done by the same team. The article meticulously details the technical approach and is accompanied by an outstanding video that should be seen by any surgeon considering this approach. The authors are completely transparent about what type of cases were done as well as the procedural and postprocedural outcomes. All valves were conventional stented valves with no sutureless valves. It should be noted that 5 of 20 $(25 \%)$ were mechanical valves and preference for a mechanical valve was an exclusion from the randomized trials. Also, 5 of 20 (25\%) had primary aortic insufficiency and 10 of $20(50 \%)$ were bicuspid valves, which were both exclusions in the randomized trials. We cannot tell from the paper where or how these numbers might overlap, but the majority 
of cases were likely have been excluded from the randomized trials.

With these caveats noted, how did the authors do with rAVR? The results in this small group were outstanding. At 30 days, there was no mortality, stroke, renal failure, or paravalvular leak. Only 1 patient had a blood transfusion, and 18 of $20(90 \%)$ were extubated in the operating room. One patient $(5 \%)$ required a pacemaker, and median length of stay was 4.5 days with almost all patients discharged directly to home. The advantage of TAVR in the low-risk randomized trials occurs mainly as a safety signal and rapid recovery in the first 30 days and these results seem to narrow or eliminate that early gap in safety and early recovery. Areas in which surgery has usually done better and are more likely to effect longer-term outcomes, such as paravalvular leak and pacemakers, are similar in this trial to standard surgery outcomes.

It is important for both the surgical and the cardiology communities to understand that these randomized trial outcomes apply only to the populations tested. Badhwar and colleagues have nicely highlighted several of the areas not tested that remain knowledge gaps at the current time for TAVR. Our program runs both active SAVR and TAVR programs and believe in both when used properly. The mean age in the low-risk trials was about 74 years of age, with only about $7 \%$ younger than 65 years of age. Bicuspid valves were excluded, and there are observation bicuspid studies but no randomized trials from which we can draw information. Planned concomitant (other than coronary artery bypass) procedures were excluded from the trial. One low-risk trial accepted only patients with access acceptable for transfemoral TAVR. ${ }^{6}$ Although the protocol exclusion for coronary artery disease was a Syntax $<32^{6}$ or $<22,{ }^{7}$ the actual level seen in the trials was much lower than this. All cases were initially screened by local experienced heart teams and deemed anatomically and physiologically to meet all inclusion and exclusion protocol criteria and to be good candidates to randomize in the trial. Despite this, $34 \%$ in one trial ${ }^{6}$ and $14.8 \%$ in the other ${ }^{7}$ were eliminated at the national screening committee level, showing the patient cohorts used to be highly selected. None of these other than concomitant procedures are likely to be exclusions to rAVR.

If all of this is true, then why are there not more rAVRs currently being done? The simple answer is that it is new and takes a very dedicated team willing to put in the time and effort seen by Badhwar and colleagues. We believe cardiac surgeons have the knowledge and skill to replicate this experience if done properly. For rAVR teams to be successful, they must fulfill the Badhwar Laws of Robotics:

First Law: rAVR must be learned and practiced outside of the operating room before clinical cases.

Second Law: rAVR takes a dedicated and consistent team.

Third Law: rAVR teams must be transparent and show safety and efficacy outcomes at least equal to standard AVR while approaching the faster recovery of TAVR.

Badhwar and his team have met all of these requirements, and we salute them on this outstanding achievement.

\section{References}

1. Cribier A, Eltchaninoff H, Bash A, Borenstein N, Tron C, Bauer F, et al. Percutaneous transcatheter implantation of an aortic valve prosthesis for calcific aortic stenosis: first human case description. Circulation. 2002;106:3006-8.

2. Smith CR, Leon MB, Mack MJ, Miller DC, Moses JW, Svensson LG, et al. Transcatheter versus surgical aortic-valve replacement in high-risk patients. $N$ Engl J Med. 2011;364:2187-98

3. Adams DH, Popma JJ, Reardon MJ, Yakubov SJ, Coselli JS, Deeb GM, et al Transcatheter aortic-valve replacement with a self-expanding prosthesis. $N$ Engl J Med. 2014;370:1790-8.

4. Leon MB, Smith CR, Mack MJ, Makkar RR, Svensson LG, Kodali SK, et al. Transcatheter or surgical aortic-valve replacement in intermediate-risk patients. N Engl J Med. 2016;374:1609-20.

5. Reardon MJ, Van Mieghem NM, Popma JJ, Kleiman NS, Sondergaard L, Mumtaz M, et al. Surgical or transcatheter aortic-valve replacement in intermediate-risk patients. N Engl J Med. 2017;376:1321-31.

6. Mack MJ, Leon MB, Thourani VH, Makkar R, Kodali SK, Russo M, et al. Transcatheter aortic-valve replacement with a balloon-expandable valve in low-risk patients. N Engl J Med. 2019;380:1695-705.

7. Popma JJ, Deeb GM, Yakubov SJ, Mumtaz M, Gada H, O'Hair D, et al. Transcath eter aortic-valve replacement with a self-expanding valve in low-risk patients. $N$ Engl J Med. 2019;380:1706-15.

8. Badhwar V, Wei LM, Cook CC, Hayanga JWA, Daggubati R, Partho P, et al. Robotic aortic valve replacement. J Thorac Cardiovasc Surg. 2021;161:1753-9. 